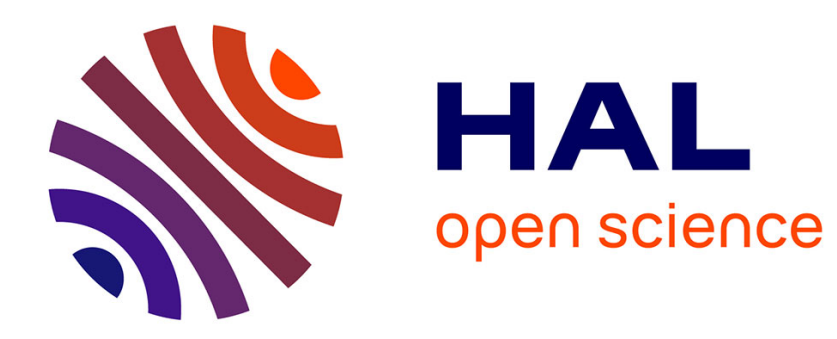

\title{
La divinité h-KTBY/hn-'KTB en Arabie du Nord-Ouest et en Jordanie du Sud
}

Saba Farès-Drappeau

\section{To cite this version:}

Saba Farès-Drappeau. La divinité h-KTBY/hn-'KTB en Arabie du Nord-Ouest et en Jordanie du Sud. Topoi Orient - Occident, 1999, 9, pp.201-208. 10.3406/topoi.1999.1815 . halshs-01736791

\section{HAL Id: halshs-01736791 https://shs.hal.science/halshs-01736791}

Submitted on 18 Mar 2018

HAL is a multi-disciplinary open access archive for the deposit and dissemination of scientific research documents, whether they are published or not. The documents may come from teaching and research institutions in France or abroad, or from public or private research centers.
L'archive ouverte pluridisciplinaire HAL, est destinée au dépôt et à la diffusion de documents scientifiques de niveau recherche, publiés ou non, émanant des établissements d'enseignement et de recherche français ou étrangers, des laboratoires publics ou privés. 


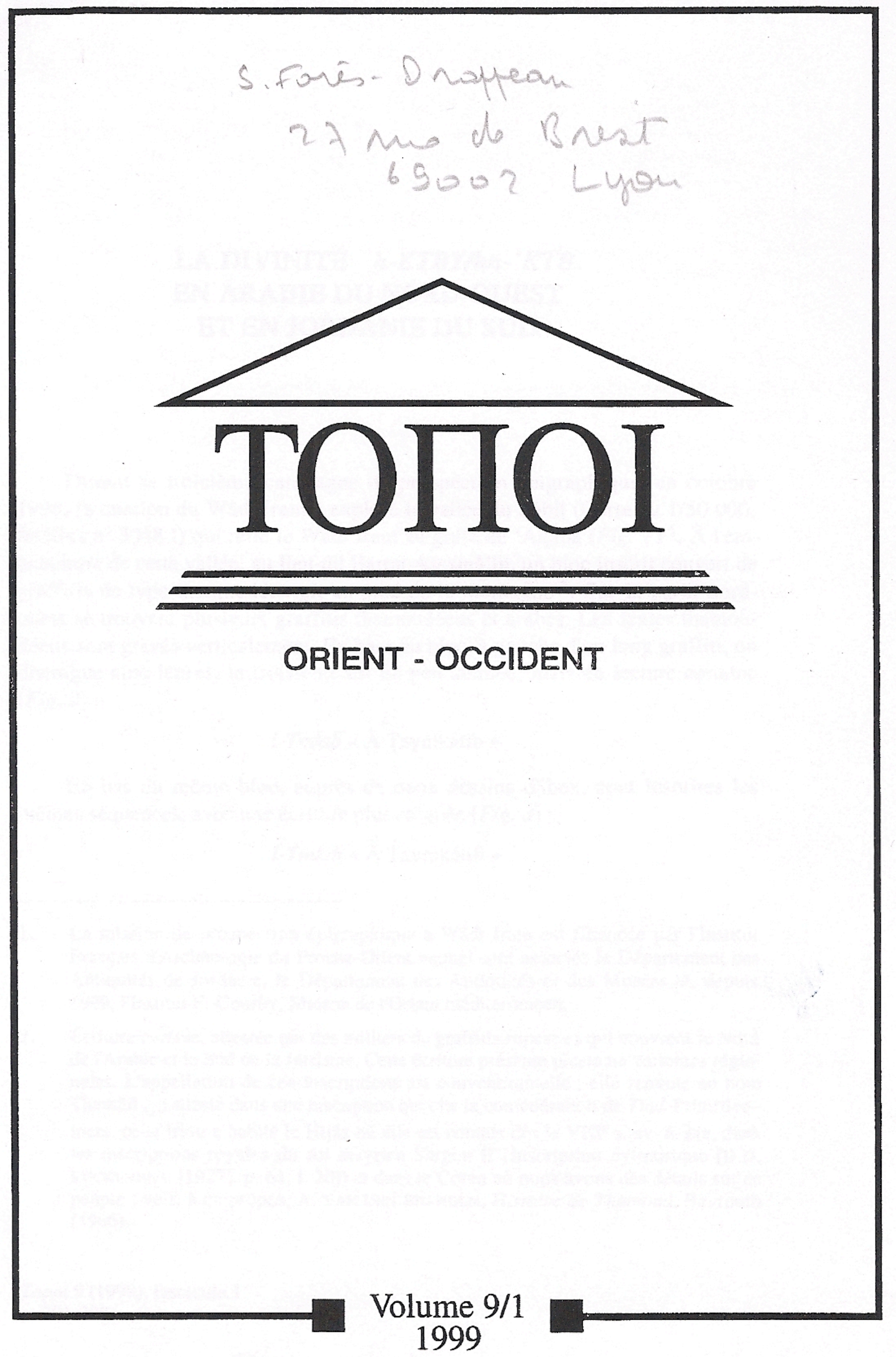




\section{LA DIVINITÉ $\quad h-K T B Y / h n$-'KTB $^{-}$ EN ARABIE DU NORD-OUEST ET EN JORDANIE DU SUD}

Durant sa troisième campagne de prospection épigraphique, en octobre 1998, la mission du Wādi Iram a exploré la vallée du Șābiṭ (Carte au 1/50 000, feuilles $n^{\circ} 3048$ I) qui relie le Wādi Iram au golfe de 'Aqaba (Fig. 1) ${ }^{1}$. À l'embouchure de cette vallée, au lieu-dit Barrat Assamā'in, un bloc inscrit couvert de graffitis de type thamoudéen gît au pied de la montagne ${ }^{2}$. Sur la paroi nordouest se trouvent plusieurs graffitis thamoudéens et arabes. Les textes thamoudéens sont gravés verticalement. En haut du bloc, à gauche d'un long graffiti, on distingue cinq lettres, la troisième est un peu abîmée, mais de lecture certaine (Fig. 2):

\section{l-Tmktb « À Taymkātib »}

En bas du même bloc, auprès de deux dessins d'ibex, sont inscrites les mêmes séquences, avec une écriture plus soignée (Fig. 3) :

\section{l-Tmktb « À Taymkātib »}

1. La mission de prospection épigraphique à Wādĩ Iram est financée par l'Institut Français d'Archéologie du Proche-Orient auquel sont associés le Département des Antiquités de Jordanie, le Département des Antiquités et des Musées et, depuis 1999, l'Institut F.-Courby, Maison de l'Orient méditerranéen.

2. Écriture cursive, attestée par des milliers de graffitis rupestres qui couvrent le Nord de l'Arabie et le Sud de la Jordanie. Cette écriture présente plusieurs variantes régionales. L'appellation de ces inscriptions est conventionnelle ; elle renvoie au nom Thamūd attesté dans une inscription qui cite la confédération de Tmd. Primitivement, cette tribu a habité le Hijāz où elle est connue dès le VIII ${ }^{\mathrm{e}} \mathrm{s}$. av. n. ère, dans les inscriptions royales du roi assyrien Sargon II (Inscription cylindrique [D.D. LUCKENBILL [1927], p. 61, 1. 20]) et dans le Coran où nous avons des détails sur ce peuple ; voir, à ce propos, A. VAN DEN BRANDEN, Histoire de Thamoud, Beyrouth (1966).

Topoi 9 (1999), fascicule 1

p. 201-208 
Jusqu'à présent cet anthroponyme théophore, dont la signification est « esclave de Kātib», était surtout attesté dans les graffitis nabatéens à Pétra et à Madā'in Șālih, sous la forme : Tymktb' ${ }^{3}$. On connaît une attestation en safaïtique : Tymhn'ktb ${ }^{4}$. Ce graffiti offre une nouvelle graphie de ce nom, il se rapproche de l'arabe en utilisant le participe actif du verbe KTB, qui correspond à kätib كاتب, dont la signification est « écrivain, scribe».

Le deuxième élément $K t b$ est un nom divin, qui entre dans la composition des anthroponymes. Il apparaît dans un nom théophore d'une inscription sudarabique trouvée dans les Émirats Arabes Unis (ash-Shāriqa) : $S^{2} m t k t b y$ (RobinMulayha 1) ${ }^{5}$. Il est surtout connu dans les inscriptions dédanites d'al-'Ulā comme nom divin et dans les noms théophores ${ }^{6}$.

Lors de la découverte des inscriptions en 1909-1914, par Jaussen et Savignac, on ignorait l'existence de cette divinité. Ces savants remarquent cependant que le nom $h n$ ' $k t b$ « paraît être une appellation divine ou le nom d'un héros divinisé [...]. Peut-être serait-il possible d'expliquer l'origine de ce mot, qui signifie "serviteur de l'écrivain" en arabe هَنى كاتب, par la vénération qu'on aurait eue

3. J.T. MILIK et J. STARCKY, «Inscriptions récemment découvertes à Pétra », ADAJ 20 (1975), p. 116-119.

4. D.L. HARDING, An Index and Concordance of Pre-Islamic Arabian Names and Inscriptions (Near and Middle East Series 8), Toronto (1971). L'écriture safaïtique est attestée par des milliers d'inscriptions en Syrie du Sud et en Jordanie du Nord. Elles doivent leur nom à la région du Șafã où les premiers textes ont été trouvés. Généralement ces inscriptions sont datées de l'époque romaine ( $\mathrm{I}^{\mathrm{e}}-\mathrm{III}^{\mathrm{e}} \mathrm{s}$. de n. ère). Les premières inscriptions ont été découvertes par Cyril Graham en 1858. Le déchiffrement a été entrepris par J. Halévy en 1877 et terminé par E. Littmann en 1901 (à ce propos, voir M.C.A. MACDONALD, «Inscriptions, Safaitic », The Anchor Bible Dictionary III [1992] p. 418-423; «S Safaitic», Encyclopaedia of Islam 8 [1994], p. 760-762; «Al-Safā », Encyclopaedia of Islam 8 [1994], p. 756-757; [1996], p. 435).

5. Chr. RobIN, « Documents de l'Arabie Antique III », Raydān 6 (1994), p. 80-81.

6. On appelle ces inscriptions « dédanites » d'après le toponyme antique Dédan (actuelle Khirbat al-Khurayba, qui se trouve à $4 \mathrm{~km}$ au nord d'al-'Ulà en Arabie du Nord-Ouest). Elles sont au nombre de quelques centaines de textes, gravés soit sur des biocs, soit sur la paroi de la montagne. Elles se trouvent dans la région qui s'étend entre al- 'Ulã et Madâ’in Șālih (environ $340 \mathrm{~km}$ au nord-ouest de Médine). Elles furent découvertes pour la première fois par Ch. Doughty en 1879 (M.Ch. Doughty, Travels in Arabia Deserta 1, réimpression New York [1979], p. 176), mais relevées surtout par A. JAUSSEN et R. SAVIGNAC, qui ont enregistré environ 380 textes: Mission archéologique en Arabie (mars-mai 1907) (Publication de la Société des fouilles archéologiques), I. De Jérusalem au Hedjaz-Médaine-Saleh, Paris (1909); II. El-'Ela, d'Hégra à Teima, Harrah de Tebouk, Paris (1914); III. Atlas, Paris (1914). La graphie des inscriptions est de forme géométrique apparentée au sudarabique, alors que la langue est très proche de l'arabe classique. La langue diffère de l'arabe par l'emploi de l'article ; elle utilise l'article $h n$-devant les gutturales 'ayn, hamza et parfois le qäf. Le $n$ étant assimilé à la consonne qui suit, on trouve généralement l'article sous la forme $h$ - 
jadis pour un homme connaissant l'art de l'écriture ${ }^{7}$. Strugnell fut le premier à l'identifier, en $1959^{8}$. Celui-ci, en réexaminant l'inscription nabatéenne de 'Ayn ash-Shallala $\left(\mathrm{n}^{\circ} 17\right)^{9}$, dont la lecture du dernier mot était imprécise, découvre qu'il s'agit d'un nom de nature divine ${ }^{10}$. Ceci l'incite à étudier deux autres inscriptions nabatéennes, provenant d'Égypte, où le nom apparaissait, mais dont la nature était restée inconnue : celle de Tall ash-Shuqayfiyya ${ }^{11}$ et celle d'un autel du petit temple de Qașr Ghayt ${ }^{12}$. Il découvre qu'il s'agit dans ces trois textes d'un même nom divin offrant à chaque fois la même graphie : ' $l k t b$ ' 13 . Reprenant une remarque de Winnett, Strugnell montre l'existence de ce nom divin en dédanite (lihyaanite) et propose un parallèle entre cette divinité et Nabu, le dieu babylonien des scribes ${ }^{14}$.

À la lumière de ces nouvelles découvertes, Milik et Teixidor reconnaissent cette divinité dans un texte nabatéen trouvé à Pétra : $q d m k w t b$ ' ' $l h$ ' $d n h$ « devant

7. A. JAUSSEN et R. SAVIGNAC, op. cit. II, p. 403.

8. J. STRUGNelL, «The Nabataean Goddess al-Kutba" and her Sanctuaries », BASOR 156 (1959), p. 29-36.

9. Publiée par R. SAVIGNAC, « Le sanctuaire d'Allat à Iram (suite)», RB XLIII (1934), p. $574-575$.

10. L'inscription est gravée sur le rocher au-dessous d'une double stèle. Elle se compose d'une seule ligne. Ce texte a été découvert en 1934 par SavignaC (ibid., p. 575), qui lit : ' $l r t b$ ' dy bgy' ' $l$ ' $z y$; il rapproche le premier mot de l'arabe rataba « être ferme, solide " et suggère d'y voir « une appellation divine devenue par la suite un nom divin comme ' $l$ ' $z y$ ». Ce mot a été lu ensuite par J. Starcky et J.T. Milik, 'ln nșb, qui traduisent le texte ainsi : « Ceux-ci sont: l'idole qui est à Gaia (et) al-'Uzza » (J.T MILIK, « Nouvelles inscriptions nabatéennes », Syria XXXV [1958], p. 247, $\mathrm{n}^{\circ} 2$ 2). J. STRUGNELL (op. cit. [1959], p. 31) a corrigé 'lktb' dy bgy' "Al-Kutbā' who is in Gaia ».

11. Cette inscription très mutilée a été trouvée dans le Delta Oriental, à l'ouest d'Ismā'siliyya. Elle comprend huit lignes. (Ch. CleRMONT-GANNEAU, «Les Nabatéens en Egypte», Recueil d'Archéologie Orientale VII [1906], p. 229-257). L'inscription est datée de la septième année $(75 / 74)$ du règne de Ptolémée XII Aulète (80-51 av. n. ère). Le nom de la divinité est abîmé, mais a été restitué par Strugnell, qui lit : (1) [dnh by]t' d[d bnh X] (2) [br ..] bw l'lktb' '[lht'] (3) ['l]hyy mr'n syw 'pkl' (4) [whlyy npšh wdy yhwh šm [h] (5) [dk]yr qdmyh wb'wytw (6) bšlm b21 lphnšy dy[šs] (7) [nt] 4 ltlmy mlk'dy hy [šn] (8) [t 1]lmr'n syw 'pkl šlm : «This is the temple (?) which $\mathrm{X}$ son of $\mathrm{Y}$ built for Al-Kutbä', the goddess, for the life of our lord Seyô, the priest, and for his own life, and in order that his name be rememberd in her presence and in 'wytw. Peace. On the 21 st of Pahonsi in the fourth year of Ptolemy the King, which is the first (?) year of our lord Seyô, the priest » (STRUGNELL, op. cit., p. 31-32).

12. J. CLÉDAT, « Fouilles à Qasr-Gheit (mai 1911)», ASAE XII (1912), p. 145-168, pl.
I-III.

13. J. STRUGNELL, op. cit., p. 29.

14. Ibid., p. 35 . 
Kutbā, ce dieu » ${ }^{15}$. Ce texte permet d'établir la prononciation Kutbā et d'assurer sa nature masculine, alors que al-Kutbā dans la littérature syriaque est une divinité féminine ${ }^{16}$.

La découverte de l'anthroponyme Tamktb dans un texte de type thamoudéen m'a amenée à réexaminer les textes dédanites où le théonyme $h n-k t b^{\prime} / h$ Ktby apparaissait. Car, si depuis la découverte de Strugnell la lecture de ces textes a été rectifiée, à ma connaissance, aucune étude n'a traité de son culte chez les Dédanites.

Le nom divin ' $l-K t b$ ' s'écrit en dédanite de deux manières : soit $h n-' k t b$, soit $h$-Ktby. Dans $h n$-' $k t b, h n$ - est l'article dédanite conservant le $n$ devant les gutturales : le hamza et le 'ayn ${ }^{17}$. La seconde graphie $h$-Ktby est la réplique exacte de la graphie nabatéenne où l'article nabatéen ' $l$ correspond à l'article dédanite $h-$, le $n$ étant assimilé devant les consonnes. Quant au hamza nabatéen final, il correspond au y finale dédanite; tous deux étant les matres lectionis pour noter le son $\bar{e}$, équivalent du alif maqsura en arabe.

Les inscriptions dédanites où le nom $h n$-' $^{\prime} t b$ et $h$-Ktb apparaît sont au nombre de trois : deux dédicaces et un texte citant le nom d'un prêtre au service de $h n$-'ktb.

- La première dédicace est un fragment de la base d'une stèle votive ou d'un petit autel. Les caractères sont soignés, la première ligne est abîmée. On lit $: h$ $m h r w-l-H n^{\prime} k t b f-r d y-h m y=$ «..] et à $H n^{\prime} k t b$. Alors Il a été satisfait d'eux deux $" 18$.

- La deuxième dédicace est gravée sur une ancienne stèle avec des caractères réguliers, un peu effacés. Le texte cite : $N[\ldots]$ h (b)nt Qsm hdqt h-șlmn

15. D.T. MLIK et J. TEIXIDOR, « New evidence on the North-Arabic deity AktabKutbâ », BASOR 163 (1961), p. 22-24. Le graffito se trouvait à l'intérieur d'un triclinium circulaire décrit par G. DALMAN, Petra und seine Felsheiligtümer, Leipzig (1908), p. 243.

16. Dans la littérature syriaque, une homélie attribuée à Méliton de Sardes (début du III ${ }^{\mathrm{e}}$ siècle de $\mathrm{n}$. ère) dit "The Mesopotamians adored KWTBY, a Hebrew (goddess)». La phrase été reprise par Théodore Bar Koni (VIII ${ }^{\mathrm{e}}$ siècle de n. ère) qui écrit «The Mesopotamians (beth nahrîn) worshipped KWTBY, an Arabian (goddess) ». Pour Milik et Teixidor cette dernière homélie confirme l'origine plutôt arabe de la divinité al-Kutbà (MILIK et TEIXIDOR, op. cit., [1961], p. 24). À propos de cette divinité, voir F. ZAYADINE, «The God(ess) Aktab-Kutbay and his (her) Iconography », in F. ZAYADINE (ed.), Petra and the Caravan Cities, Proceedings of the Symposium organised at Petra in September 1985 by the Department of Antiquities of Jordan and the Iconographic Lexicon of Classical Mythology (LIMC), Amman (1990), p. 37-51.

17. En dédanite, l'article est $h n$-. Le $n$ est assimilé à la lettre qui le suit, sauf devant les gutturales hamza, 'ayn, et, parfois, le qăf.

18. A. JAUSSEN et R. SAVIGNAC, op. cit. II, nº 37 (lihyānite), p. 366-367, pl. LXXIX. 
l-Hn'ktb f-rd-h $w$-'hrrt-h = « [...] fille de Qsm a offert la statue à $H n^{\prime} k t b$. Alors II a été satisfait d'elle et l'a guidée ${ }^{19}$.

- Enfin, le nom apparaît dans le texte suivant : ... Jy wkl $h$-Ǵsn 'fkl $h$-Ktby

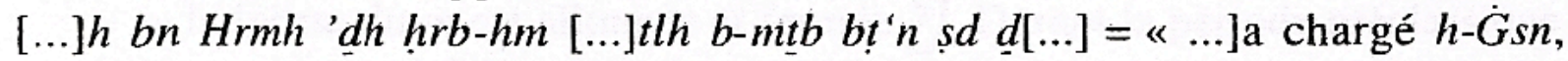
prêtre de $h$-Ktby [...] fils de $H r m h$, lorsqu'il leur fit la guerre [...] à $m \underline{t} b . . .{ }^{20}$.

Deux noms théophores composés avec $h n$-' $k t b$ sont attestés également en dédanite : Zdhn'ktb, Zaydhan'aktub «accroissement de han'aktub » ${ }^{21}$, et $M r m h n ' k t b$, «Désir de han'aktub » 22.

Les diverses formes $h n$-' $k t b, h-K t b y, ' l-K t b$ ' et $K t b$ rappellent la racine sémitique KTB, dont la signification est «écrire». Ceci évoque, pour Strugnell, Nabu ${ }^{23}$, dieu des scribes, de l'écriture et de la sagesse, l'un des principaux dieux du panthéon babylonien ${ }^{24}$. À l'époque néo-babylonienne on observe un renouveau de ferveur à l'égard de ce dieu. Le séjour, d'environ dix ans, du roi Nabonide ( ca 553-543) à Taymä' a probablement été à l'origine de la diffusion de son culte dans la région 25 .

La datation précise des inscriptions dédanites est difficile à établir. La graphie de ces textes étant la même, la chronologie relative, fondée sur l'analyse pa-

19. A. JAUSSEN et R. SAVIGNAC, op. cit. II, $\mathrm{n}^{\circ} 62 / 1-6$, p. 402-403; estampage pl. LXXXIII, copie pl. CVI.

20. A. JAUSSEN et R. SAVIGNAC, op. cit. II, n 55/1, p. 394-395, estampage pl. LXXXII, copie pl. CV. Le mot 'fkl est d'origine akkadienne $a b$-(p)kallu, qui a le sens de « sage ». Kitāb al-Aghānī cite le nom d'un personnage 'Amrū ibn Ju'ayd عمر بن جعيد qui était prêtre kāhin sاهol. XV, p. 76). Ce même personnage esț cité chez lbṇn Durayd, dans al-Ishtiqāq, portant le titre de al-afkal «كل (Al-Ištiqāq, 'Abd asSalām Muhammad Hārūn édition, 2 vol., Bag̉dād (éd. al-Muthannà) [1979], p. 325), il était connu pour être tyrannique. D'après A. Jaussen et R. Savignac, il est probable qu'en arabe la signification d'afkal «crainte, tremblement » dérive de ce personnage (JAUSSEN et SAVIGNAC, Mission Archéologique en Arabie Il [1914], p. 385-386.

21. A. JAUSSEN et R. SAVIGNAC, op. cit., $n^{\circ} 78 / 1$, p. 445-446, estampage pl. LXXXVIII, copie pl. CXII ; n 358/2, p. 527, pl. CXXXIX.

22. A. Jaussen et R. SAVignaC, op. cit., $\mathrm{n}^{\circ} 290$, estampage pl. XCIII et copie pl. CXXXVI.

23. J. STRUGnell (op. cit., p. 35) propose d'établir des parallèles avec Nabu, le dieu babylonien des scribes, l'égyptien Toth et le dieu des Grecs et des Romains, Hermès-Mercure.

24. J. BOTTÉRO, La religion babylonienne, Paris (1952), p. 41-42.

25. Nabonide, dernier roi babylonien, était fils d'un gouverneur et d'une prêtresse du dieu Sîn de Harrān. Il est connu dans l'histoire pour avoir quitté Babylone et séjourné environ dix ans à Taymā', en Arabie du Nord-Ouest (ca 553-543). Les raisons de ce séjour demeurent obscures et ont fait l'objet d'un long débat (R.P. DouGHERTY, Nabonide and Belshazzar, New-Haven [1929], p. 105-117, 138-160 ; C.J. GADD, «The Harran Inscriptions of Nabonidus », AnSt VIII [1958], p. 76-89 ; W.G. LAMBERT, « Nabonidus in Arabia », PSAS 1971 [1972], p. 53-64 ; P.-A. BEAULIEU, The Reign of Nabonidus, King of Babylon 556-539 B. C. [1989], p. 149-232). 
léographique, les situe durant la période classique des inscriptions, c'est-à-dire approximativement au $\mathrm{V}^{\mathrm{e}}-\mathrm{IV}^{\mathrm{e}} \mathrm{s}$. av. n. ère. Les lettres durant cette période graphique ont les caractères suivants :

- la forme du $m$ est allongée et fermée 2 . Durant la période préclassique le $m$ a deux triangles $\varangle$, comme en sudarabique, alors que durant la période récente il a une forme allongée et ouverte vers le bas $\}$.

- Les parallèles horizontales des $b$, 'et le $k$ sont légèrement convergentes

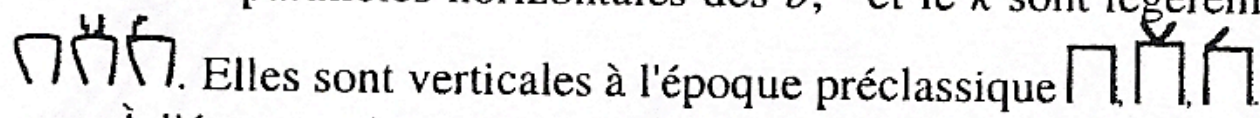

À l'époque récente le $b$ ne change pas, alors que les parallèles $k$ forment un

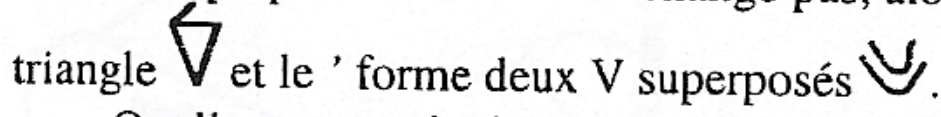

Quelle que soit la datation exacte de ces textes, ils sont vraisemblablement antérieurs aux inscriptions nabatéennes qui ne remontent pas au delà du IIe siècle av. n. ère.

Les Nabatéens ne semblent pas avoir pris d'importance avant le III ${ }^{\mathrm{e}}$ siècle av. n. ère. À cette date, ils ont dû parvenir à supplanter la tribu de Lihyân et à rassembler les tribus arabes dans une nouvelle confédération, qui est la leur ${ }^{26}$. L'une des tribus, qui dépendait de la confédération de Lihyān et qui vénérait $h$ $K t b y$ / ' $l-K t b$ ', a probablement conservé le culte de $h-K t b y$ / ' $l-K t b$ ' sous les Nabatéens, c'est ce qui explique probablement sa diffusion restreinte. Cette divinité n'était le dieu tutélaire ni de la confédération de Lihyyān ni des Nabatéens ; elle était vénérée probablement par une tribu dépendante de l'ancienne confédération. Le culte a continué sous la nouvelle confédération, jusqu'au milieu du I $^{\text {er }}$ siècle de n. ère, si l'on se fonde sur la graphie des inscriptions de Iram et de Pétra, qui ne suggèrent pas une datation plus tardive ${ }^{27}$. À partir de cette date, d'autres divinités arabes apparaissent, telle qu'al-Lāt. Celle-ci, mentionnée par Hérodote dès le $\mathrm{V}^{\mathrm{e}} \mathrm{s}$. av. n. ère ${ }^{28}$, devient la divinité arabe la plus importante à l'époque romaine ( $\mathrm{I}^{\mathrm{e}}$-IIIJ $\mathrm{S}$. de $\mathrm{n}$. ère) ${ }^{29}$.

Saba FARÈS-DRAPPEAU

26. Lihyān, cité dans la titulature royale des inscriptions dédanites trouvées à Khirbat al-Khurayba (cf. n. 6), est un nom ethnique, connu comme anthroponyme dans les inscriptions sudarabiques (cf. M. ARBACH, Répertoire des noms propres du Corpus Inscriptionum Semiticarum, Pars Quarta inscriptiones Himyariticas et Sabceas continens, thèse d'État, Aix-en-Provence [1993]). Pendant longtemps les savants ont distingué « inscription dédanite » et « inscription lihyānite », deux termes employés dans le titre royal.

27. J. STARCKY, « Pétra et la Nabatène », Dictionnaire de la Bible VII (1966), col. 996.

28. Hérodote, Histoires, III, 8 ; I, 131.

29. J. STARCKY, op. cit., col. 1001. 


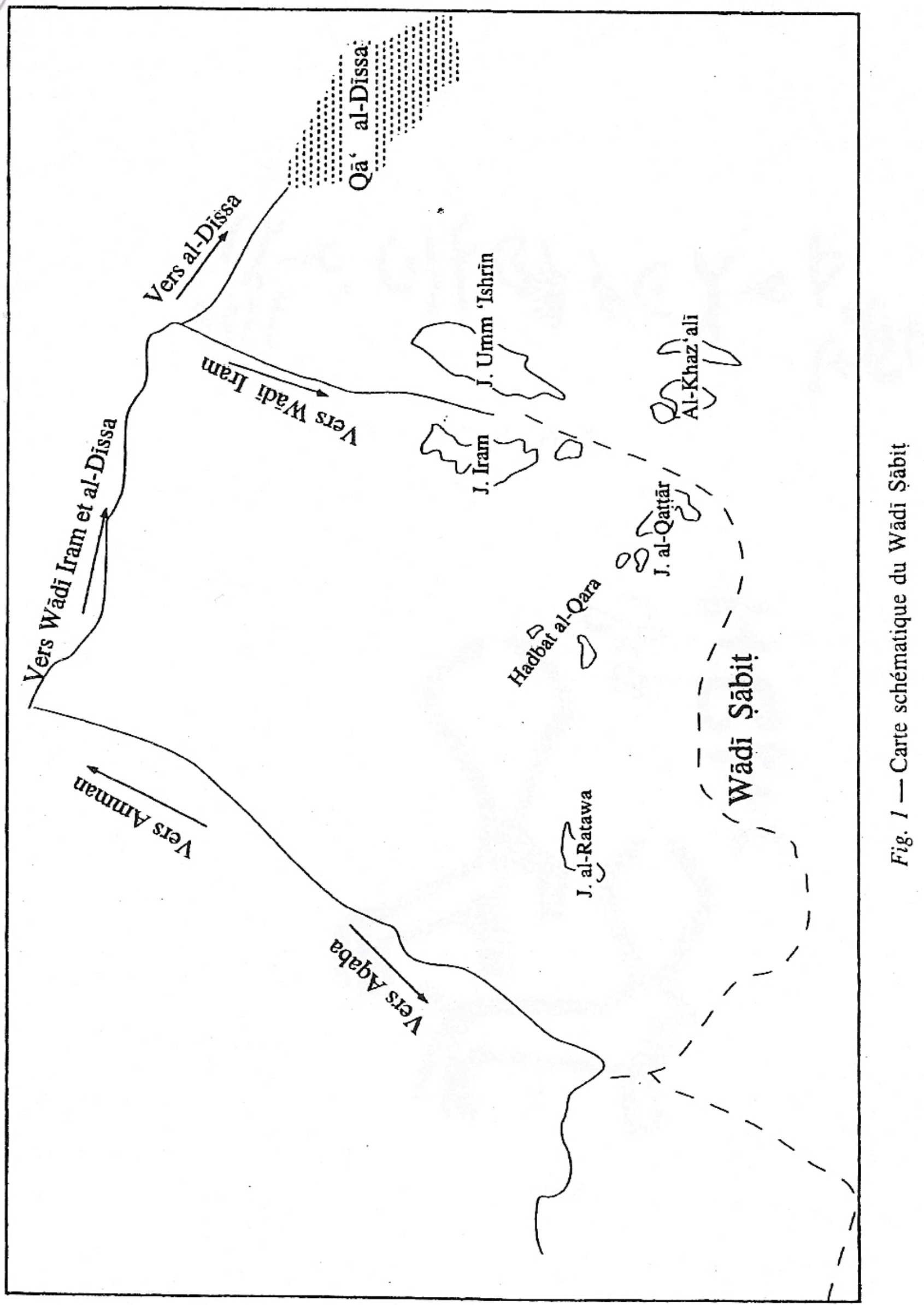




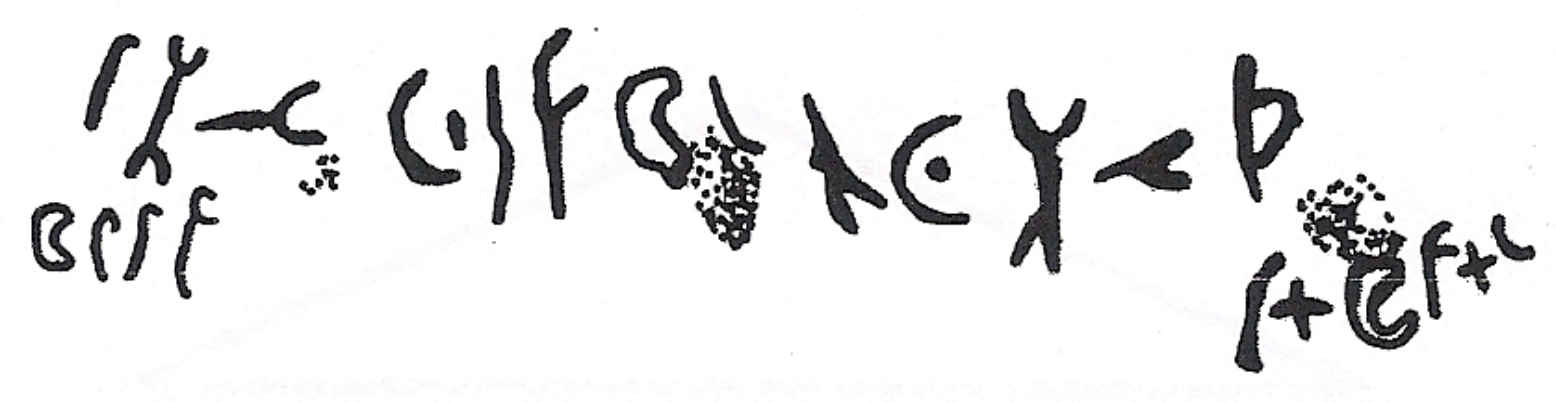

Fig. 2

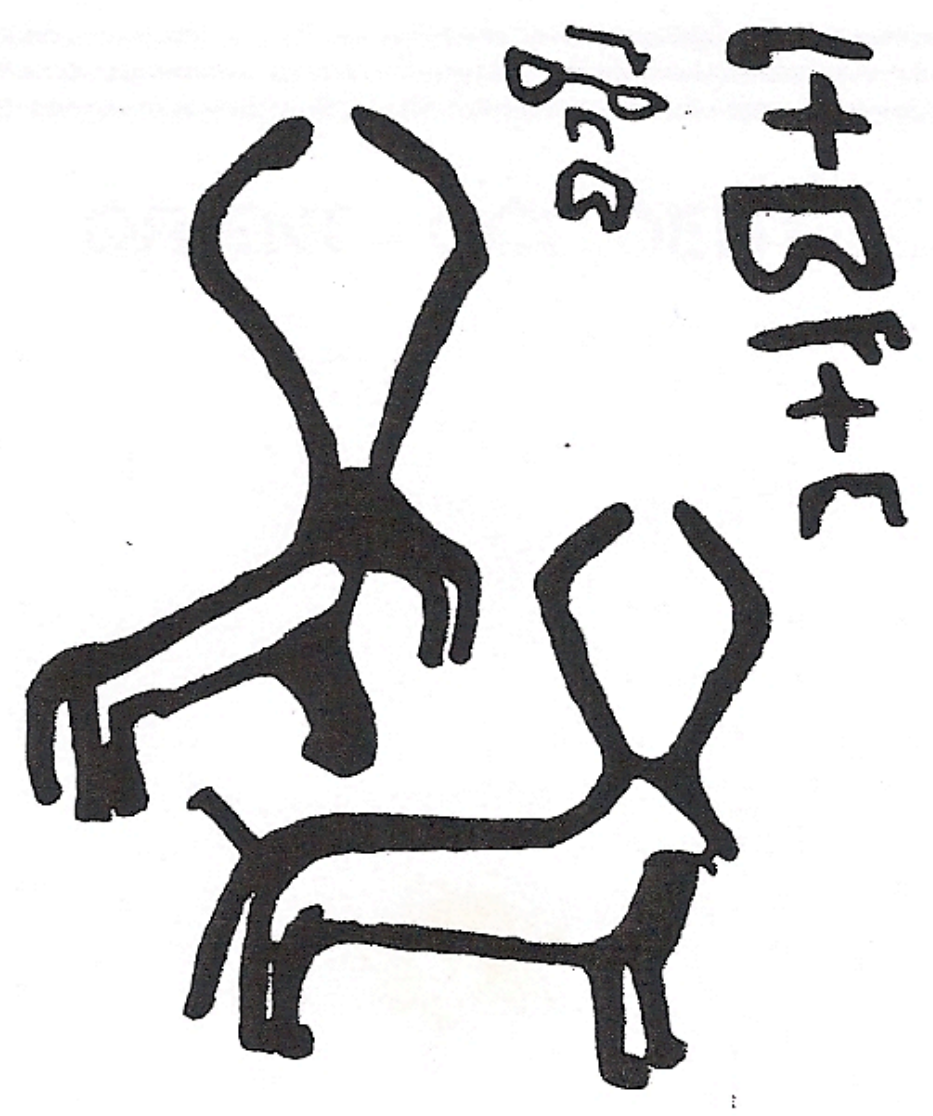

Fig. 3 